\title{
Enhancing Forensic-Tool Security with Rust: Development of a String Extraction Utility
}

Jens Getreu

Taltech, Tallinn University of Technology

Olaf Maennel

Taltech, Tallinn University of Technology

Follow this and additional works at: https://commons.erau.edu/jdfsl

Part of the Computer Law Commons, and the Information Security Commons

\section{Recommended Citation}

Getreu, Jens and Maennel, Olaf (2019) "Enhancing Forensic-Tool Security with Rust: Development of a String Extraction Utility," Journal of Digital Forensics, Security and Law. Vol. 14 : No. 2 , Article 4. DOI: https://doi.org/10.15394/jdfsl.2019.1607

Available at: https://commons.erau.edu/jdfsl/vol14/iss2/4

This Article is brought to you for free and open access by

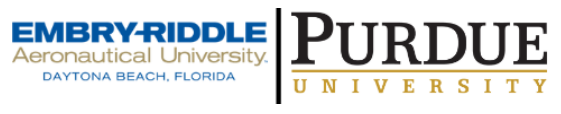
the Journals at Scholarly Commons. It has been accepted for inclusion in Journal of Digital Forensics, Security and Law by an authorized administrator of Scholarly Commons. For more information, please contact commons@erau.edu.

(c)ADFSL 


\title{
ENHANCING FORENSIC-TOOL SECURITY WITH RUST: DEVELOPMENT OF A STRING EXTRACTION UTILITY
}

\author{
Jens Getreu, Olaf Maennel \\ Taltech, Tallinn University of Technology \\ Ehitajate tee 5 \\ 19086 Tallinn, Estonia
}

\begin{abstract}
The paper evaluates the suitability of the Rust ecosystem for forensic tool development. As a case study, a forensic tool named Stringsext is developed. Starting from analyzing the specific requirements of forensic software in general and those of the present case study, all stages of the software development life-cycle are executed and evaluated.Stringsext is a reimplementation and enhancement of the $G N U$-strings tool, a widely used program in forensic investigations. Stringsext recognizes Cyrillic, CJKV East Asian characters and other scripts in all supported multi-byte-encodings while $G N U$-strings fails in finding these in UTF-16 and other encodings. During the case study it has become apparent that the Rust ecosystem provides good support for secure coding principles and unit testing. Furthermore, the benchmarks showed a satisfactory performance of the resulting Stringsext binaries comparable to the original $C$ version.
\end{abstract}

Keywords: Forensic analysis, string search, multi-byte encoding, Rust language, Stringsext-tool

\section{INTRODUCTION}

Human interaction with electronic devices leaves traces in their electronic memory. In the age of cloud computing most human interaction triggers requests to distant servers leaving traces not only in their log files, but also in many intermediate network devices. Due to the cross-linked nature of computer systems the data that needs to be taken into consideration when investigating a crime is enormous. In this huge amount of information the investigator has to find those specific bits of information constituting digital evidences. In the domain of digital forensics an electronic trace (observation) with a wellknown cause-effect-relationship between the observation and the human action causing it (activity), is a so called artefact. For the sake of simplicity we present here a rather simplistic view associating univocally one trace (artefact/observation) with only one possible cause/activity. In the physical world, one trace might have several possible causes which cannot be excluded a priori. For this reason, modern forensics favors the Likelihood Ratio approach, in which the degree of support of the observations for the hypotheses is considered, providing a strength of evidence (diagnostic value) that 
is relative to the hypotheses tested. It embodies any "item of interest that helps an investigation move forward." (Harichandran, Walnycky, Baggili, \& Breitinger, 2016, p. $125)^{1}$.

Forensic examiners, the law enforcement personnel who deal with digital evidence, face inter alia two challenges: to collect and to preserve the huge amount of data that may be related to a crime and to search and detect artifacts in the collected data. The latter aspect implies the so called string search which is useful when dealing with unknown binary data. Most binary data contain human readable character sequences called strings. A very commonly used program to extract strings from a binary data is the so called $G N U$-strings program. Although still widely used, GNU-strings has only a very rudimentary support for multibyte encodings such as Unicode. Furthermore, it was subject several to memory safety vulnerabilities which exclude the handling of potentially harmful forensic data. The software tool Stringsext, developed in this present work, is made for same purpose. The new development is designed to overcome both shortcomings. Where possible, it maintains $G N U$-strings' user-interface.

In the following section we analyze general tool requirements in digital forensics. Together with the shortcomings of the original GNU-strings tool, it becomes obvious that the $\mathrm{C}++$ programming language does not satisfy the indispensable security requirements in the field of forensic software. The remaining sections show how the Rust-

${ }^{1}$ Cf. Harichandran (Harichandran et al., 2016, p. 131) who proposed a more formal definition: A Curated (digital) Forensic Artefact (CuFA) "must have evidentiary value in a legal proceeding, must be created by an external force/artificially, must have antecedent temporal relation/importance and must be exceptional (based on accident, rarity, or personal interest) $[\ldots] "$. language mitigates them and what should be observed during implementation.

\section{TOOL REQUIREMENTS IN DIGITAL FORENSICS}

\subsection{Multi-byte character encoding support}

Like in other established forensic disciplines the forensic soundness and reliability of digital evidence depend on the validity and correctness of the forensic software used in examination. In other words, to guarantee that the digital evidence is forensically sound, all tools used to collect, preserve and analyze digital evidences must be validated. Tool validation can also be formally required by standards like the ISO 17025 Laboratory Accreditation standard.

Validation is the confirmation by examination and the provision of objective evidence that a tool, technique or procedure functions correctly and as intended (Craiger, Swauger, Marberry, \& Hendricks, 2006, p. 92).

One way of establishing a set of requirements for a new forensic tool is to analyze how similar existing tools are validated. Beckett and Slay (Beckett \& Slay, 2007) propose a functionality oriented validation method called Model of tool neutral testing. Instead of testing if a software product meets all its requirements, an independent set of forensic functions is defined and later tested. The digital forensic discipline can be broadly defined in terms of the key functions Identification, Data Preservation, Data Analysis and Presentation of Digital Evidence. Each key function is further divided into subcategories. For example Data Analysis 
breaks down into: Searching, File Rendering, Data Recovery, Decryption, Identification, Processing, Temporal Data and Process Automation. The first item searching relates to finding and locating information of interest in digital memories. Form a functional point of view the searching falls into the searching domain (where), the searching mode (how) and the searching target (what). This breakdown of forensic functions into detailed categories allows to decouple the validation procedure from the implementation of the forensic tool itself. Based on this categorization, independent test environments are set up for each specific function. For example, a test may certify if the software is able to run a fuzzy search for strings in the unallocated disk space.

Beyond validation, the categorization of forensic functions is also helpful to define requirements for improvement existing tools - in our case GNU strings. For example, Searching can be further classified into groups as shown in the Figure 1 (Beckett \& Slay, 2007, p. 17). The leaves in the diagram list typical capabilities a Searching-software can implement. For example the subcategory "Character encoding" illustrates the main deficit of GNU-strings as it supports only ASCII encoding. In a global cyberspace, forensic tools must identify a multitude of encodings. This leads us to the main motivation and requirement of Stringsext: multibyte character encoding support.

The functionality oriented validation can be classified as "black box testing" examining functionality without any knowledge of the internal implementation, even without having access to the source code. Its advantage is, that it allows to reuse the test environment thus reducing costs. Black box testing is sometimes also referred as "behavioral testing" as it feeds the tool with known test case data and observes if the tool outputs the expected results. In the context of this work black box testing is used to assert that the developed tool Stringsext deals correctly with big real-world input data: Stringsext has been designed to produce, as a special case, bit-identical output to that of $G N U$ strings. This way the comparison of the output data of both tools allowed to confirm that stringsext is working correctly when dealing with big data.

In addition to the above black box testing, Rust's build in test harness (unit test) is used in conjunction with the test driven development in order to guarantee maximum security and correctness of the developed tool.

\subsection{Security}

"Make it hard for them to find you and impossible for them to prove they found you". With this concise word Berinato (2007) characterizes the relation between the criminal and the forensic investigator. In digital forensics this "hide-and-seek" game might soon take a new dimension: Eggendorfer (2016) stresses with good reasons that forensic tools are software too and therefor vulnerable to attacks: A vulnerability found in 2017 in a common forensic tool EnCase Forensic Imager demonstrates exemplary the pertinence of the risk (Consult, 2017):

By writing a manipulated LVM2 partition (a hard disk format commonly used for Linux servers) on a storage device, an attacker could - if the device were ever to be analyzed using EnCase Forensic Imager - take over an investigator's machine. When the investigator tries to read the device, EnCase Forensic Imager crashes - unbeknownst to the investigator, however, a lot more is happening. Through a buffer overflow security 


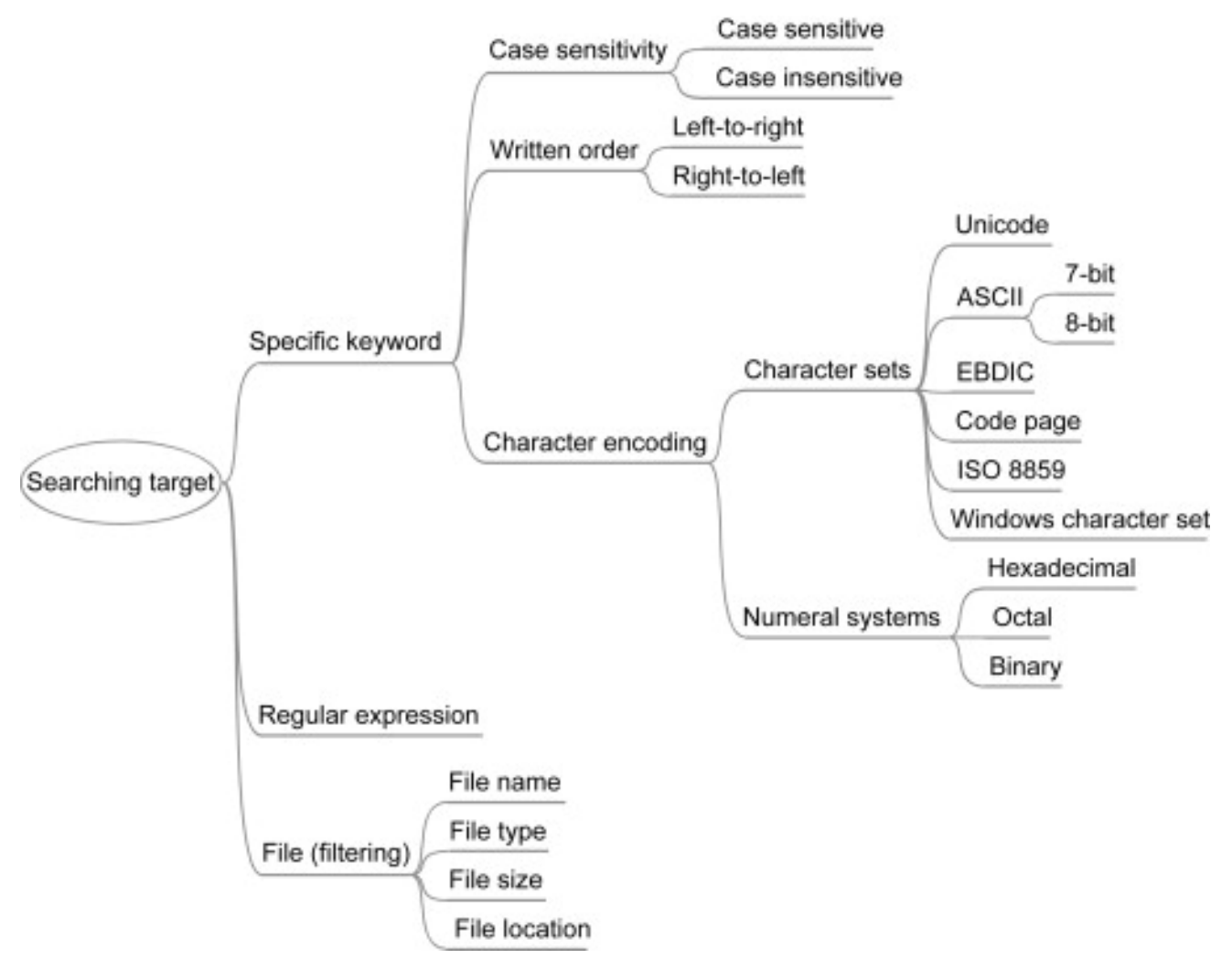

Figure 1. The search target mapping, cf. (Beckett \& Slay, 2007, p. 8)

flaw, EnCase Forensic Imager can be tricked into executing data read from the storage device. Afterwards the code provided by the attacker has full control of the investigator's machine and can be used by the suspect to manipulate evidence (Consult, 2017).

Confronted with this vulnerability report, the vendor downgraded the issue: "The exploit SEC Consult claims to have found is an extreme edge case, much like the theoretical alerts they tried to promote in November. [...] We do not consider this claim to be serious and it will not impact the performance of our products (Consult, 2017)." For the user it remains unclear if and when the vulnerability gets fixed. While such a reaction would have been inconceivable in other software domains, the risk of forensic tool exploitation is still largely neglected despite its potential impact:

- The adversary may be warned about an ongoing investigation.

- The adversary may gain control of the investigator's machine and alter evidences.

TheStringsext-project addresses this risk by choosing the programming language Rust. Rust offers some outstanding security guarantees which are presented in Section 4 .

\subsection{Code efficiency}

The searching domain in forensic investigations is often as large as the seized datacarrier. Nowadays hard-disk images hold several TiB of data. Memory images of the RAM are smaller, but still some GiB in size. In order to address so big search domains, forensic software must operate very efficiently. This is why forensic software 
is often programmed in $\mathrm{C}$ or $\mathrm{C}++$. But not only the programming language matters: Efficient code also requires carefully chosen abstractions, efficient algorithms avoiding unnecessary data-copies and programloops. Concerning the choice of the programming language we define the following requirements: The programming language should:

- allow a fine control over pointers and memory allocation,

- offer zero cost abstractions,

- have no or a minimal runtime system.

The above motivates the choice of developing Stringsext in Rust. Chapter 4 shows how Rust meets the above requirements by its memory safety guarantees and zero cost design goal.

\section{GNU-STRINGS SHORTCOMINGS IN FORENSICS}

This section first analyzes GNU-strings' limitations concerning multi-byte-encodings and international scripts. Based upon this we derive a set of additional requirements for Stringsext. Many forensic practitioners use the GNU program strings, hereafter referred as GNU strings, to get a sense of the functionality of an unknown program by extracting human readable strings from binary data. Of special interest are for example strings containing URLs to malicious sites, often an indicator of malware activity. But also, user prompts, error messages, and status messages can give valuable hints.

\subsection{International character encodings}

As discussed above the main motivation for developing Stringsext is the missing multi- byte character encoding semantics in $G N U$ strings. GNU-strings encoding support consists of a rudimentary filter accessed with the option--encoding. Please consult the manual page for more details. How well does $G N U$-strings detect Unicode? The Figure 2 shows the content of a text file chosen as test case. To find out how well $G N U$

\begin{tabular}{|c|c|}
\hline $\begin{array}{l}\text { Arabic: } \\
\text { Chinese: }\end{array}$ & حبل الكذب قدير \\
\hline French: & Les pâtes \\
\hline Greek: & Ioторía \\
\hline German: & Viele Grüße \\
\hline Russian: & Поздравляю \\
\hline Symbols: & $€, \quad$ \\
\hline
\end{tabular}

Figure 2. Test case international character encodings

Strings deals with different Unicode encodings, the text-file is then is converted into UTF-8, UTF-16LE, UTF-16BE, UTF-32LE and UTF-32BE, each encoding in one file. In order to observe $G N U$-strings Unicode detection capabilities, all the test-files are then searched for valid graphic strings with the command strings using all possible variation of its encoding filter. The Figure 3 shows exemplary GNU-strings output for a UTF-16 little endian encoded input.

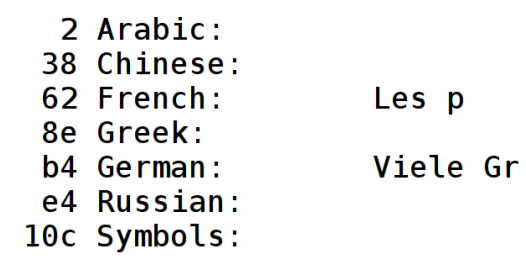

Figure 3. GNU-strings with UTF-16LE encoded input

Results: UTF-8 is the only encoding in which $G N U$ strings is able to find international characters. The Figure 3, chosen as an example, shows that with UTF-16 input, $G N U$ strings fails to recognize all non-ASCII characters. The same holds true for UTF-32 and most other encodings: This limitation is 
of particular importance in forensic investigations: The Microsoft-Windows operating system handles Unicode characters in memory as 2 byte UTF-16 words. As a result when dealing with Microsoft-Windows memory images, $G N U$-strings is not able to detect any international characters! It should not be forgotten that $G N U$-strings is not designed to analyze multi-byte encodings in general. This is why other very common encodings e.g. big5 or kois-r are not supported at all even though they are widely used. The above-outlined limitations leads to Stringsext's main requirement: character encoding support.

\subsection{Secure coding}

In the narrow sense, "secure coding" is rather a design goal than a functional requirement. Secure coding denotes the practice of developing computer software by reducing the accidental introduction of security vulnerabilities by preventing coding errors or discovering and eliminating security flaws during implementation and testing. From the secure coding point of view the requirement character encoding support is the most critical: The NIST National Vulnerability Database lists under the heading "character encoding" 22 vulnerabilities. Not only new complex forensic software is affected by vulnerabilities. It also concerns other wellestablished products: The tool GNU-strings, part of the $G N U$ binutils collection, became publicly available in 1999 (Cygnus-Solutions, 1999). Today it has reached the notable age of 17 years. GNU-strings is a comparatively small program with 724 lines of code only. It is all the more surprising that in 2014 the security researcher Zalewski (2014) discovered a serious security vulnerability CVE-20148485 :

\section{The setup group function in bfd/elf.c in libbfd in GNU}

binutils 2.24 and earlier allows remote attackers to cause a denial of service (crash) and possibly execute arbitrary code via crafted section group headers in an ELF file.

Zalewski headlined his bug report "Don't run strings on untrusted files." Needless to say that this advice can not be followed in the context of a forensic investigation. In the meantime the bug was fixed but users remain confused and bewildered.

Of particular importance is that the above bugs are part of a vulnerability class related to memory safety problems. GNU strings is written in $\mathrm{C}$, a language whose abstractions can not guarantee memory safety. In order to exclude potential vulnerabilities of the same kind from the outset, Stringsext was developed in the Rust programming language.

\section{THE RUST PROGRAMMING LANGUAGE}

In the Section 2 we showed that the requirements code efficiency and security are of paramount importance. This section illustrates how Rust supports these requirements with its zero cost abstractions and its guaranteed memory safety (The-Rust-Team, 2019) motivating the choice of implementing Stringsext in Rust.

\subsection{Memory safety}

All memory-related problems in $C$ and $C++$ come from the fact that $C$ programs can unrestrainedly manipulate pointer to variables and objects outside of their memory location and their lifetime. The Table 1 shows a selection of most common memory safety related vulnerabilities (Corporation, 
2016). Memory safe languages like Java do not give programmers direct and uncontrolled access to pointers. For example, in Java memory safety is guaranteed through a resource costly runtime and a garbage collector. The related additional costs in terms of runtime resources exclude programming language like Java for most forensic tool development.

For many years program efficiency and memory safety seemed to be an insurmountable discrepancy. Now, after 10 years of development, a new programming language called Rust promises to cope with this balancing act. Rust's main innovation is the introduction of semantics defining data ownership. This new programming paradigm allows the compiler to guarantee memory safety at compile-time. Thus, no resource costly runtime is needed for that purpose. In Rust most of the weaknesses listed in Table 1 are already detected at compile time. Moreover, the Rust compiler guarantees that none of these weaknesses can result in an undefined system state or provoke data leakage.

Rust's main innovation is the introduction of new semantics defining ownership and borrowing. Put in simplified terms, they translate to the following three rules which Rust's type system enforces at compile time:

1. All resource (everything that can be bound to a variable, e.g. integers, vectors, structures) has a unique owner.

2. Others can borrow from the owner (technically borrowing means that another scope sets a pointer to the owner's resource).

3. The owner cannot free or mutate the resource while it is borrowed.

By enforcing the above rules Rust organizes how resources are shared among different scopes. Memory problems occur for instance

\begin{tabular}{|ll|}
\hline CWE ID & Name \\
\hline \hline 119 & $\begin{array}{l}\text { Improper Restriction of Operations } \\
\text { within the Bounds of a Memory } \\
\text { Buffer }\end{array}$ \\
\hline 120 & $\begin{array}{l}\text { Buffer Copy without Checking Size } \\
\text { of Input ('Classic Buffer Overflow') }\end{array}$ \\
\hline 125 & Out-of-bounds Read \\
\hline 126 & Buffer Over-read ('Heartbleed bug') \\
\hline 122 & Heap-based Buffer Overflow \\
\hline 129 & Improper Validation of Array Index \\
\hline 401 & $\begin{array}{l}\text { Improper Release of Memory Before } \\
\text { Removing Last Reference ('Memory } \\
\text { Leak') }\end{array}$ \\
\hline 415 & Double Free \\
\hline 416 & \begin{tabular}{l} 
Use After Free \\
\hline 591
\end{tabular} \\
\hline 763 & $\begin{array}{l}\text { Sensitive Data Storage in Improp- } \\
\text { erly Locked Memory } \\
\text { ence }\end{array}$ \\
\hline
\end{tabular}

Table 1. Common weaknesses in $\mathrm{C} / \mathrm{C}++$ that affect memory

when a resource is referenced by multiple pointers (aliasing) and when more than one pointer writes to the same memory at the same time (mutation). In contrast to other languages, Rust's semantics allow the type system to ensure at compile time that simultaneous aliasing and mutation mutually exclude each other (cf. Table 2). As the check is performed at compile-time, no run-time code is necessary. Furthermore, Rust does not need a garbage-collector: when owned data goes out of scope it is immediately destroyed.

The following code samples (The-RustProject-Developers, 2017, Sec. 3.2) illustrate how the Rust compiler detects nonobvious hidden memory safety issues. The function as_str returns a pointer to a stack allocated resource $\mathbf{s}$ that is freed at the end of the function: we find ourselves with a "Use after free" condition. The compiler aborts with the error message $s$ does not live long enough. 


\begin{tabular}{|l|l|l|l|}
\hline $\begin{array}{l}\text { Resource } \\
\text { sharing }\end{array}$ & $\begin{array}{l}\text { Alias- } \\
\text { ing }\end{array}$ & $\begin{array}{l}\text { Muta- } \\
\text { tion }\end{array}$ & Example \\
\hline \hline $\begin{array}{l}\text { move } \\
\text { owner- } \\
\text { ship }\end{array}$ & no & yes & let a=b \\
\hline $\begin{array}{l}\text { shared } \\
\text { borrow }\end{array}$ & yes & no & let $\mathrm{a}=$ \&b \\
\hline $\begin{array}{l}\text { mutable } \\
\text { borrow }\end{array}$ & no & yes & let $\mathrm{a}=$ \&mut $\mathrm{b}$ \\
\hline
\end{tabular}

Table 2. Resource sharing in Rust

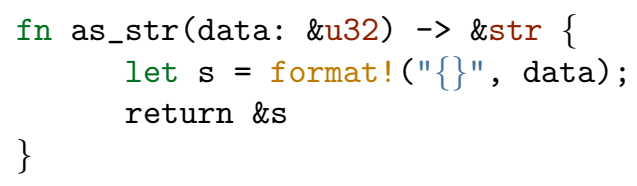

Here the corrected memory safe code performing a byte-copy at the end of the function.

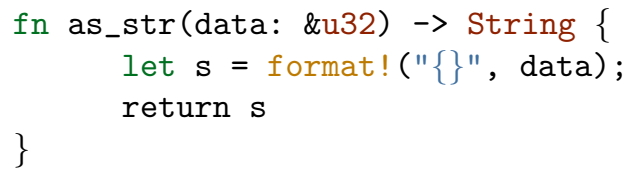

The push() method in line 3 of the next example causes the backing storage of data to be reallocated. As a result we have a dangling pointer vulnerability! Again, the Rust compiler detects the error and code does not compile.

let mut data $=\operatorname{vec} ![1,2,3]$;

let $\mathrm{x}=$ \&data $[0]$;

data. push (4);

println! (" \{\}$", \mathrm{x})$;

Here the corrected memory safe version that compiles:

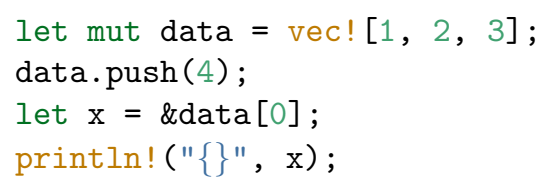

\subsection{Iterators}

A very common group of programming mistakes is related to improper handling of indexes especially in loops, e.g. "CWE-129:
Improper Validation of Array Index" (cf. Table 3 (Corporation, 2016)).

\begin{tabular}{|l|l|}
\hline CWE ID & Name \\
\hline \hline 119 & $\begin{array}{l}\text { Improper Restriction of Operations } \\
\text { within the Bounds of a Memory } \\
\text { Buffer }\end{array}$ \\
\hline 125 & Out-of-bounds Read \\
\hline 129 & Improper Validation of Array Index \\
\hline
\end{tabular}

Table 3. Common weaknesses in $\mathrm{C} / \mathrm{C}++$ affecting memory avoidable with iterators (Corporation, 2016)

In addition to traditional imperative loop control structures, Rust offers efficient iteration with functional style iterators. Like in Haskell iterators are lazy and avoid allocating memory for intermediate structures (you only allocate just when you call . collect()). Iterators considerably enhance the robustness and safety of programs. They enable the programmer to iterate through vectors without explicitly naming neither the index nor its bounds, thus avoiding common mistakes. The Figure 4 shows an example.

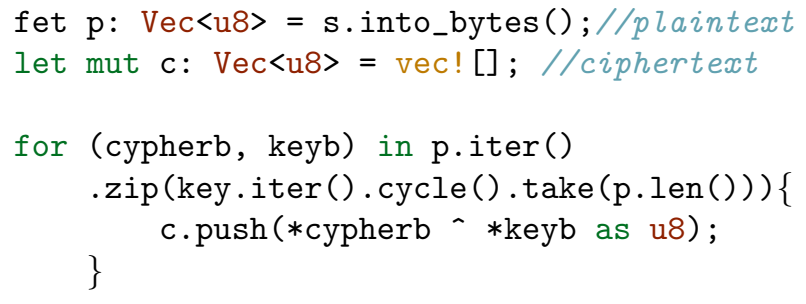

Figure 4. Vigenère cipher in Rust

\subsection{Zero-Cost Abstractions}

It is the language design goal Zero-Cost $A b$ stractions that makes the $\mathrm{C} / \mathrm{C}++$ language so efficient and suitable for system programming. It means that libraries implementing abstractions, e.g. vectors and strings, must be designed in a way that the compiled binary is as efficient as if the program had been 
written in Assembly. This is best illustrated with memory layouts: Figure 5 shows a vector in Rust. Its memory layout is very similar is to a vector in $C++$. In contrast, the

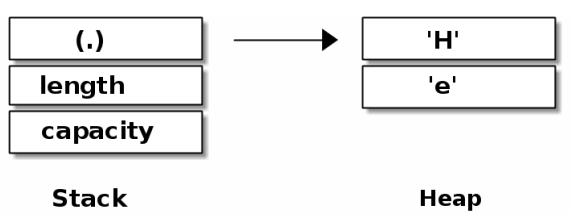

Figure 5. Memory layout of a Rust vector

memory safe language Java enforces a uniform internal representation of data. In Java a vector has 2 indirections instead of 1 compared to Rust and $C / C++$ (cf. Fig. 6). As the data could be represented in a more efficient way in memory, we see that Java does not prioritize Zero-Cost-Abstraction as primary objective.

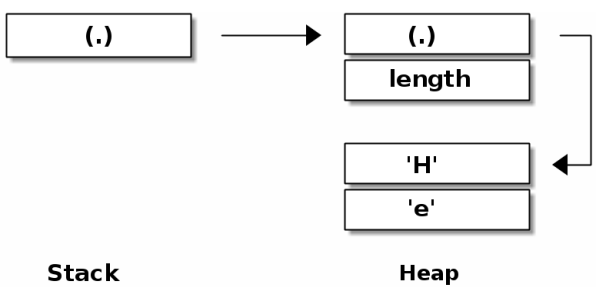

Figure 6. Memory layout of a Java vector

\section{USE CASE: DEVELOPMENT OF THE STRINGSEXT-TOOL}

In the Section 2 we have analyzed the special requirements for tools in digital forensics: code efficiency and security. The Section 4 showed that Rust's core properties zero cost abstractions and memory safety in theory meet well our requirements.

But how well is the Rust ecosystem suited for forensic tool development? In order to evaluate also the practical aspects of Rust, the tool Stringsext (Getreu, 2018)(Getreu, 2017) was developed. The technical challenges such as concurrent batch processing of multi-byte character streams revealed to be sufficiently complex, thus allowing us to deduce general recommendations for forensictool development.

\subsection{Encoding support}

The initial motivation for developing Stringsext were the various shortcomings of $G N U$ strings especially when it comes to handle international character encodings. Does Stringsext support foreign scripts better? Is it as fast?

To evaluate Stringsext's capabilities to handle international scripts with Unicode, we choose the same input text file (cf. Figure 2) we used with GNU-strings in the Section, 3.1. Stringsext's output (cf. Figure 7) confirms that all international characters are found correctly. Furthermore, Figure 8 illustrates how Stringsext's formats its output when it operates in simultaneous multiencoding scanning mode.

Stringsext's unique Unicode range restriction feature has shown itself to be of use in practice: The Microsoft-Windows operating system handles Unicode characters in memory as 2 byte UTF-16 words. Thus, searching for UTF-16 encoded strings in memory images is common practice in forensics. This is more difficult as expected, as almost every random byte combination is assigned to a valid Unicode code point. Without further measures such a search leads to many false positives and unusable results. The offered solution by Stringsext allows the user to restrict the Unicode-range: For example, the Unicode-range U+400-U+7FF captures only strings in Cyrillic, Armenian, Hebrew, Arabic and Syriac. This feature is particularly useful when dealing with UTF-16 in memory images. 


\begin{tabular}{|c|c|c|}
\hline $\begin{array}{r}0 \text { (utf-16le) } \\
\text { (utf-16le) } \\
\text { (utf-16le) } \\
\text { (utf-16le) } \\
\text { (utf-16le) } \\
\text { (utf-16le) } \\
\text { (utf-16le) }\end{array}$ & $\begin{array}{l}\text { Arabic: } \\
\text { Chinese: } \\
\text { French: } \\
\text { Greek: } \\
\text { German: } \\
\text { Russian: } \\
\text { Symbols: }\end{array}$ & 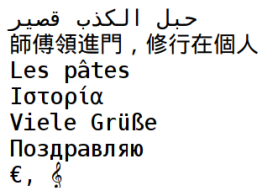 \\
\hline
\end{tabular}

Figure 7. Stringsext's output with UTF-16LE encoded input

\begin{tabular}{|c|c|c|}
\hline$\odot$ & (utf-16be) & c 遭武献晡琀 $\mathrm{I}$ À \\
\hline$\odot$ & (utf-16le) & 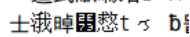 \\
\hline & (utf-16le) & 士涐晫用慗 $\mathrm{t} s$ 똩 \\
\hline 47 & (ascii) & NO NAME \\
\hline 47 & (ut f-8) & NO NAME \\
\hline 77 & (ascii) & This is not a \\
\hline & (ascii) & press any key \\
\hline 77 & $\begin{array}{l}\text { (ut } f-8) \\
\text { (ut } f-\beta)\end{array}$ & $\begin{array}{l}\text { This is not a } \\
\text { press any key }\end{array}$ \\
\hline 3ea & (ut f-16be) & ᄃ 遭武献晡琀 İ Ä \\
\hline c47 & (ascii) & NO NAME \\
\hline c47 & (utf-8) & NO NAME \\
\hline c77 & $\begin{array}{l}\text { (ascii) } \\
\text { (ascii) }\end{array}$ & $\begin{array}{l}\text { This is not a } \\
\text { press any key }\end{array}$ \\
\hline c77 & (ut $f-8$ ) & This is not a \\
\hline & (ut $f-8)$ & press any ke \\
\hline & (ut f - 16be) & 及/闻／《／間／ \\
\hline & 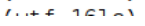 & \\
\hline
\end{tabular}

Figure 8. Stringsext's output in multiencoding scanning mode

\subsection{Concurrency}

The Figure 9 shows the data flow in Stringsext. All scanner instances as well as the merger-printer are designed as threads. Rust uses OS-level threads and its type and ownership model guarantees the absence of data races. Rust supports by default two models of inter-thread communication: shared memory and message channels.

Stringsext first cuts the input stream into overlapping slices of shared read-only memory pages, which are then fed into the different scanner threads. Each scanner operates independently in batch. It runs through the input-slice, searches for valid string sequences, collects them in a list and finally sends the list to a merger-printer-thread though a dedicated message channel. This thread collects all lists from the different scanners and merges them into the final output stream.

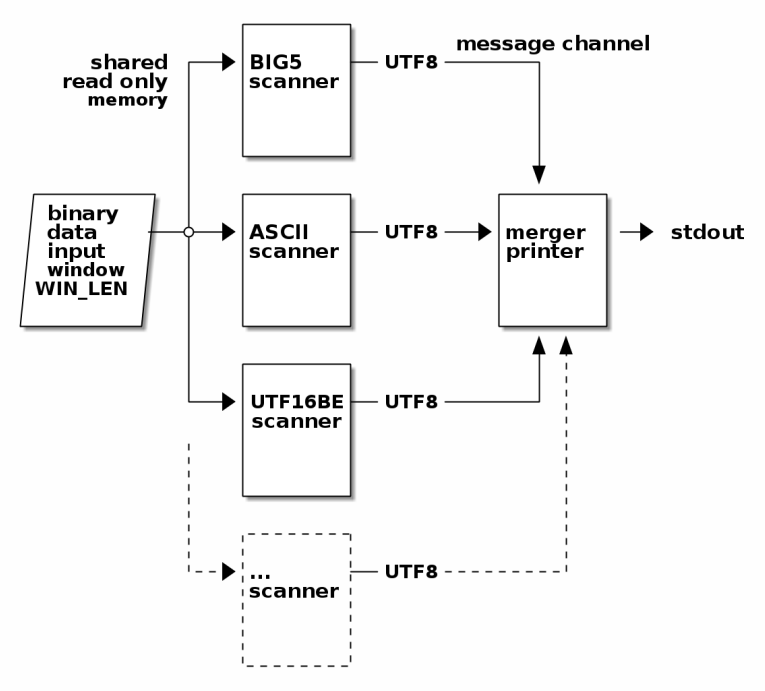

Figure 9. Data processing and threads

\subsection{Algorithm}

Batch processing of multibyte character streams turned out to be more difficult than expected. As we want to keep the scanning process as stateless as possible, we introduced overlapping windows: This allows reading beyond the memory page's edge in case a found string terminates in the next memory page. However, strings can be so long that you can't but cut them somewhere. In this case we need to make sure not to cut in the middle of a multi-byte character which can be up to 6 bytes long. As a result a scanner is not completely stateless: between each scanner run the position where the last run stopped and a flag indicating the forced cut of a string is passed.

The scanner decodes the input stream in two phases: first it uses the Encoding-crate library to identify valid code sequences and transcodes them into valid UTF-8 strings. Such a valid string may contain non-graphical and graphical characters. As we are only interested in the latter, a second filter extracts graphical substrings while meeting additional criteria, e.g. minimum 
length or Unicode-range restriction rules.

\section{DEVELOPMENT PROCESS EVALUATION}

Besides the contribution of the new tool Stringsext to the forensic community a more general consideration is of scientific interest: Seeing that Rust is a very young programming language: how well is the Rust ecosystem suited for forensic tool development?

Forensic tools have to fulfill stringent requirements concerning their quality: In general, huge amount of data has to be processed which leads to most demanding requirements in terms code efficiency (cf. Section 2.3). Furthermore, the data to be analyzed is potentially dangerous: it may contain malicious payload targeting common vulnerabilities (cf. Section 2.2). Finally, in order to fulfill legal requirements forensic tools must be extensively tested.

The present case study confirms our initial hypothesis that Rust meets these requirements: Rust, as system programming language, is designed for code efficiency. In addition Rust guarantees memory safety, the cause for a common category of vulnerabilities. It's build in unit testing feature supports software verification as defined in the Section 2.1.

Memory safety is checked at compile time by Rust's borrow checker: When a Rust source code compiles, the resulting binary is guaranteed to be memory safe. In consequence, such a binary is immune to memory safety related attacks: e.g. out-ofbounds read, buffer over-read, heap-based buffer overflow, improper validation of array index, improper release of memory before removing last, double free, use after free. As Stringsext and all its used libraries are solely Rust components, Stringsext is memory safe.
We compared the code efficiency of $G N U$ strings implemented in $\mathrm{C}$ and Stringsext implemented in Rust: When Stringsext is run in ASCII-only mode, both produce bit-identical output. The field experiment yielded that even though Stringsext's 2.4 times slower, the speed is within the same order of magnitude. However, Stringsext's design implies much more complex computations, hence the result is not surprising.

How about the efficiency of Rust's abstractions and its overall performance? A good estimation is to compare benchmarks of small and simple programs. Too complex programs should be avoided for this purpose because variations of the programmer's skills may bias the result. According to the "Computer Language Benchmark Game" (Fulgham \& Gouy, 2019) Rust and $C / C++$ have similar benchmark results, which confirms our above measurements.

Forensic tools have to operate on many architectures. Here enters Rust's crosscompiling feature on scene. As Rust uses the LLVM framework as back-end, it is available for most platforms. rustc --print target-list lists 80 compiler targets (rustc version 1.27).

As discussed above, Rust's memory safety guarantee is a huge improvement in terms of security because a whole category of potential vulnerabilities can be ruled out from the outset. However, memory safety does not necessarily mean there is no bug. Beside the security aspects discussed above, the correctness of forensic software is crucial (cf. Section 2.1). It is clear that the overall correctness of a program also depend on the correctness of every library used. Hence, the question arises whether the Rust ecosystem is mature enough to meet the ambitious requirements of forensic software. Indeed, compared to C, Rust's libraries are relatively young. Here again extensive unit testing revealed to be a helpful diagnostic method: for 
example one of the first versions 0.4 .16 of the kmerge function, part of the itertools library used in Stringsext, reversed under rare conditions the first and second finding. Unit testing revealed a bug in itertools. The programmer responded quickly. It took only some days after its appearance that the bug was fixed with pull request \#135. So far this was the only time we encountered a bug in the used libraries. One conclusion we draw from this experience is, that young libraries are more likely to have bugs than established ones. It cannot be emphasised enough that diligent unit testing helps to find most bugs at early state. However, unit testing do not help against memory safety related vulnerabilities, which are typical for $\mathrm{C}$ and $\mathrm{C}++$ programs and which can persist in software for decades. Taking into account these benefits and drawbacks we largely prefer accepting the greater likelihood of manageable bugs related to young Rust libraries, than the uncertainty of hidden memory safety related vulnerabilities typical for $C$ and $C++$.

Finally, we recognize the benefits of unit testing throughout this work. For this reason we chose for this project the test driven development method where unit testing is the key element. Contrary to other methods, in test driven development unit tests and the to be tested code is always programmed by the same person, which fitted well the setting of this project. However, other methods may be as suitable depending on the organisational structure of the programmer team.

\section{CONCLUSION}

We have shown that forensic tool development is subject to special requirements in terms of memory safety and code efficiency. Both are inherent properties of the used programming language and its ecosystem. Even though the language design of $\mathrm{C}$ and $\mathrm{C}++$ allows generating very efficient code, there is no guaranty that the compiled code is memory safe. Not only that the lack of memory safety is one of the principal causes for most software vulnerabilities, forensic software is particularly exposed to such risks as it processes binary data of unknown origin containing all kinds of malware. As an alternative to $\mathrm{C}$ and $\mathrm{C}++$, the relatively new programming language Rust offers guarantied memory safety by design, while being as fast as C. The match-making of general forensic tool requirements and the theoretical properties of the Rust programming language makes it an ideal alternative to the hitherto dominant programming language $\mathrm{C}++$. But a general recommendation for a shift in programming practises can not be deduced from theoretical considerations alone: this is why we developed the Stringsext-Software as use case.

The use case "development of the Stringsext-tool" shows that Rust was a good choice for the present project, even though batch processing of multi-bytes character streams revealed to be far more complex than expected. Additionally, concurrent programming in Rust posed a hurdle at the beginning. Fortunately, the friendly Rust community helped to overcome occasional technical obstacles quickly. In addition, for a not so experienced Rust programmer it is reassuring to know that when a complex piece of code finally compiles, it is memory safe. The same applies to common settings when a programmer has to refactor existing code. Rust clears away doubts like "Do I free the memory at the right moment? Is this pointer still valid?" Furthermore, Rust is especially suitable for bigger projects where several programmers contribute to the same code. And this is particularly true when developing forensic software with its high quality standards.

It has to be noted though that the Rust ecosystem is still very young and bugs in 
new libraries are nothing uncommon. Fortunately, the library maintainers are very responsive and a bug is usually fixed within days. Here again unit testing becomes handy. It does not only find bugs in our own code at early stage, it also helps to identity bugs in external libraries. Used together with the test driven development method, the test code and the to be tested code can be validated in one go.

Stringsext is currently in production state and can be used in forensic investigations as a $G N U$ strings replacement. It is especially useful where $G N U$-strings fails: it allows finding UTF-16 multi-byte characters in memory images and supports other multibyte encodings like big5 or kois-r.

\section{REFERENCES}

Beckett, J., \& Slay, J. (2007). Digital forensics: Validation and verification in a dynamic work environment. In System Sciences, 200\%. HICSS $200 \%$. 40th Annual Hawaii International Conference (p. 266a-266a). IEEE.

Berinato, S. (2007, June). The Rise of Anti Forensics.

http://www.csoonline.com/ article/ 2122329.

Consult, S. (2017, May). Chainsaw of Custody: Manipulating forensic evidence the easy way.

Corporation, M. (2016). CWE - Common Weakness Enumeration, a Community-Developed Dictionary of Software Weakness Types. https://cwe.mitre.org/.

Craiger, P., Swauger, J., Marberry, C., \& Hendricks, C. (2006). Validation of digital forensics tools. Digital crime and forensic science in cyberspace. Hershey, PA: Idea Group Inc, 91-105. Cygnus-Solutions. (1999, May). Log message: Sourceware import. https://sourceware.org/ ml/ binutils-cvs/ 1999-q2/ msg00000.html.

Eggendorfer, T. (2016, July). IT forensics. Why post-mortem is dead. Cyber Security Summer School 2016: Digital Forensics, Technology and Law. Tallinn University of Technology. Fulgham, B., \& Gouy, I. (2019, February). Computer Language Benchmarks Game: $C++$ versus Rust. https://benchmarksgameteam.pages.debian.net/ benchmarksgame/ faster/ rust.html.

Getreu, J. (2017). Forensic-Tool Development with Rust (Unpublished doctoral dissertation). Tallinn University of Technology, Tallinn.

Getreu, J. (2018). Stringsext, a GNU Strings Alternative with Multi-Byte-Encoding Support. Tallinn.

Harichandran, V. S., Walnycky, D., Baggili, I., \& Breitinger, F. (2016). CuFA: A more formal definition for digital forensic artifacts. Digital Investigation, 18, S125-S137.

The-Rust-Project-Developers. (2017). The Rustonomicon.

The-Rust-Team. (2019, January). Rust Documentation. https://doc.rust-lang.org/.

Zalewski, M. (2014, October). PSA: Don't run 'strings' on untrusted files (CVE-2014-8485). 